\title{
"DNA barcoding" is of limited value for identifying adelgids (Hemiptera: Adelgidae) but supports traditional morphological taxonomy
}

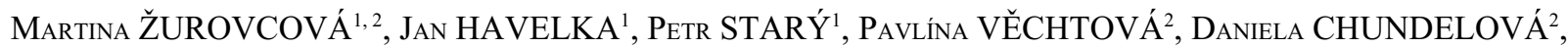 \\ ANDREA JAROŠOVÁ ${ }^{2}$ and LuCIE KUČEROVÁ ${ }^{1,2}$ \\ ${ }^{1}$ Biology Centre ASCR, Institute of Entomology, Branišovská 31, CZ-37005 České Budějovice, Czech Republic; \\ e-mail:martina@entu.cas.cz \\ ${ }^{2}$ Faculty of Science, University of South Bohemia, Branišovská 31, CZ-370 05 České Budějovice, Czech Republic
}

Key words. Adelgidae, aphids, species identification, systematics, DNA barcoding

\begin{abstract}
The sequence diversity in the mitochondrial cytochrome-c oxidase I (COI) gene was evaluated as a tool for resolving differences among species of European adelgids collected from several localities across the Czech Republic. Members of 7 genera and 16 species were examined, and as outgroups, two species of Phylloxeridae were used. Sequence divergences within species were on average less than $0.15 \%$, whereas divergences between species ranged from 0.0 to $4.12 \%$ for congeneric and to $13.24 \%$ for intergeneric comparisons. It is concluded that DNA barcoding of Adelgidae is a powerful tool for identifying genera, but at the species level it works only in those cases where there are no species complexes. Nevertheless, it can be used as a complement to traditional, morphological taxonomy.
\end{abstract}

\section{INTRODUCTION}

Adelgids are a small group of aphids, feeding on a variety of coniferous trees. With their very complex life cycles and biology (cyclical parthenogenesis, multiple generations, host-switching) they present a great taxonomical challenge. Their larval stages are morphologically almost indistinguishable, which makes species identification by traditional methods (i.e. microscopic inspection of series of individuals from the same clone) very cumbersome and time-consuming. In fact, only a very skilled professional can distinguish adelgids with certainity, which is not very practical for every day forestry management.

The classification of adelgids both at the species and genus levels is not completely satisfactory and it is more than obvious, that besides the morphological structures and bionomical details that have been studied since the end of the $19^{\text {th }}$ century, modern molecular approaches are needed.

In principle, two systems are currently used in studies of the systematics of adelgids. In the USA and UK, all the species are sorted into two genera, Adelges and Pineus. These genera are distinguished primarily by the number of abdominal spiracles in adults (Annand, 1928; Carter, 1971; Blackman \& Eastop, 1994). This system is currently used by taxonomists in the New World/UK such as Havill et al. (2007) and Havill \& Foottit (2007). However, the majority of systematicists in the Western and Eastern Palearctic regions distinguish 8 genera: Adelges, Cholodkovskya, Sacchiphantes, Dreyfusia, Aphrastasia, Gilletteella, Pineus, and Eopineus. This system is based on details of the morphology of first instar larvae (Börner, 1908, 1930, 1952; Inouye, 1945; Börner \& Heinze, 1957; Bodenheimer \& Swirski, 1957; Steffan,
1961, 1968, 1972; Heinze, 1962; Shaposhnikov, 1964; Dmitriev, 1965; Szelegiewicz, 1968; Pashchenko, 1988; Binazzi \& Covassi, 1991), alatae forms (Binazzi, 2000) as well as bionomical characteristics and morphology of the galls (Cholodkovsky, 1896; Pašek, 1954; Börner \& Heinze, 1957; Shaposhnikov, 1964; Lampel, 1968; Steffan, 1972).

Steffan (1968) introduced the use of chromosome numbers and appearance of endosymbionts, and suggested a modification of the subfamily grouping. He shifted the genera Dreyfusia and Aphrastasia to Pineinae, thus reducing the Adelginae group to only four genera (Gilletteella, Sacchiphantes, Cholodkovskya, and Adelges).

A first attempt to utilize molecular markers for adelgid systematics was that of Havill et al. (2007). The phylogenetic relationships based on mitochondrial cytochrome oxidase subunit I (COI), cytochrome oxidase subunit II $(C O I I)$, and cytochrome b (cytb) genes and the nuclear elongation factor $1 \alpha(E F-1 \alpha)$ locus were reconstructed. The results support the previous morphology-based division into two genera proposed by Annad (1928) or the two subfamilies of Börner \& Heinze (1957), respectively. However, this study focused on higher level phylogenetic classification and host specificity. Several species were represented by only one individual or not at all.

Recently, one approach to DNA-based species identification has quickly gained many supporters as well as opponents. The method is called "DNA barcoding" and is based on the assumption that variability found in the segment of cca $650 \mathrm{bp}$ of the mitochondrial gene for cytochrome oxidase subunit I (COI) is sufficient for the identification of species (Hebert et al., 2004) or even for species delimitation (Tautz et al., 2003). As the literature on "DNA barcoding" grows it is clear that these generali- 
zations do not apply across the whole animal kingdom (Vences et al., 2005; Shearer \& Coffroth, 2008), and a more careful statistical approach should be applied (Meyer \& Paulay, 2005; Meier et al., 2006, 2008; Weimers \& Fiedler, 2007).

Despite its limitations, "DNA barcoding" can nevertheless help with species identification if applied correctly. However, it is first necessary to generate a database containing the "DNA barcodes" of the target taxa and then determine whether it can be used for species identification in a pilot study. Foottit et al. (2009) did this based on a set of 17 adelgid species. They concluded that "DNA barcoding" has potential for the detection of cryptic species, but can not distinguish species defined by life-cycle characteristics.

Here the goal is to establish a "DNA barcoding database" of adelgid species found in the Czech Republic, and determine its usefulness for identification compared to using morphological and ecological characters.

\section{MATERIAL AND METHODS}

Samples were collected in 2005, 2007, and 2008 from several different localities in the Czech Republic (95 samples), Lithuania (1 sample), and Serbia (1 sample) (Table 1). Specimens (several individuals per clone) were either preserved in $100 \%$ ethanol or frozen and kept at $-70^{\circ} \mathrm{C}$ until further analysis. Several individuals of Phylloxera coccinea and Viteus vitifoliae (both Phylloxeridae) were used as an outgroup. Adelgid collections are available in the Institute of Entomology, Biology Centre ASCR, České Budějovice. Microscopic identifications were made by J. Havelka.

Total genomic DNA was extracted from 3-5 specimens per clone using two different methods. A "Quick protocol" (Frati et al., 2001) was used for the frozen samples, while the ethanol preserved samples were extracted via ZR Genomic DNA Kit (Zymoresearch Inc., Orange, CA, USA) according to the manufacturer's protocol. PCR amplification was carried out using the TaKaRa Ex Taq system (TaKaRa Bio Inc., Otsu, Shiga, Japan) or TopBio polymerase Unis (TopBio s.r.o., Prague, Czech Republic) and universal primers LCO1490 and HCO2198 (Folmer et al., 1994) to amplify cca. $670 \mathrm{bp}$ of the 5' end of mitochondrial cytochrome oxidase I ( $\operatorname{cox} 1)$. Reaction volumes $(50 \mu 1)$ consisted of $5 \mu 1$ of template DNA (not quantified), $5 \mu 1$ of $10 \times$ reaction buffer, $4 \mu 1$ of dNTP mixture $(2.5 \mathrm{mM}$ each), $0.5 \mathrm{mM}$ of each primer, and 1.25 unit of Taq polymerase. The PCR reactions were carried out in a Mastercycler ep gradient $S$ thermocycler (Eppendorf AG, Hamburg, Germany) with the following profile: $94^{\circ} \mathrm{C}$ for $1 \mathrm{~min}$ followed by 35 cycles of $94^{\circ} \mathrm{C}$ for $30 \mathrm{~s}, 47^{\circ} \mathrm{C}$ for $45 \mathrm{~s}, 72^{\circ} \mathrm{C}$ for $1 \mathrm{~min}$, and final extension at $72^{\circ} \mathrm{C}$ for $3 \mathrm{~min}$. PCR products were cleaned with either the DNA Clean\&Concentrator-5 Kit (Zymoresearch) or enzymatically with ExoSAP-IT (USB Corporation, Cleveland, Ohio, USA) before directly sequencing.

Sequencing was performed in both directions using the above primers in a BigDye v. 3.1 sequencing reaction on an ABI 310 automated sequencer (Applied Biosystems, Inc., Carlsbad, CA, USA) at the sequencing facility of the Laboratory of Genomics (Biology Centre ASCR, České Budějovice). Sequences were edited and aligned both manually and with the assistance of SeqMan (Lasergene 8 package of programs from DNASTAR, Inc., Madison, WI, USA). Morphological vouchers are kept within the collection of the Institute of Entomology, Biology Centre ASCR, České Budějovice.
Distance analysis was performed using the MEGA v.4 software (Tamura et al., 2007), which was also used to create Neighbour-Joining tree based on uncorrected $p$-distances (bootstrap analysis with 10,000 replicates) and divergence time estimates. $\chi^{2}$ test as implemented in PAUP* 4.0.b10 (2003) was used to determine the homogeneity of base frequencies among sequences. The same program and Modeltest (Posada \& Crandall, 1998) were used to select the best fitting model for further phylogenetic analysis.

To explore the sensitivity of our results to the choice of reconstruction method, we also conducted Bayesian analyses using the GTR - site specific rate model with MrBayes v3.1.2 (Ronquist \& Huelsenbeck, 2003) and maximum-likelihood (ML) analysis with PHYML (Guindon \& Gascuel, 2003). In both of these analyses the GTR + I + G model selected by the Modeltest was used. In the Bayesian analysis, $1 \mathrm{st}+2 \mathrm{nd}+3 \mathrm{rd}$ codon position were treated as separate partitions. Two different settings were explored. In the first, node support was assessed as the posterior probability from five independent runs, each with one chain of 2,500,000 generations (sampled at intervals of 100 generations with a burn-in of 6250 trees). In the second, node support was assessed as the posterior probability from two independent runs each with four chains (temperature for hot chains lowered to 0.1 ) of 10,000,000 generations (sampled at intervals of 100 generations with a burn-in of 25000 trees). For the maximum likelihood analysis, a random initial tree, best of NNI and SPR searches for the tree topology estimate, 5 independent runs and $1000 \times$ bootstrap, were used.

We also utilized TaxonDNA (Meier et al., 2006) to obtain a frequency distribution for intra- and interspecific congeneric genetic variability and evaluate the potential of the "DNA barcoding" for identifying species of adelgid. In short, this program evaluates the similarity of the species sequences, assigning those with the closest match and the same species name as successfully identified. If there are several equally good best matches from different species, identification is considered ambiguous, which applies also to the species represented by a single sequence.

\section{RESULTS}

The $687 \mathrm{bp}$ fragment of the COI gene was sequenced for 97 individuals, representing 16 species of adelgids and 2 phylloxerids. Two species, Aphrastasia pectinatae and Pineus pineoides, were represented by a single sample. No insertions, deletions, or stop codons were detected. Therefore, it is concluded that it is likely that only mitochondrial loci were sequenced and there are no nuclear pseudogenes (NUMT) in our data set. Of the 195 variable sites, 181 were parsimony informative. An alignment of the sequences is available from the authors upon request. All sequences have been deposited in GenBank (Table 1).

Nucleotide composition averaged over all adelgids showed an $\mathrm{A}+\mathrm{T}$ bias $(\mathrm{A}=36.4 \%, \mathrm{~T}=39.1 \%, \mathrm{C}=14.8 \%$, $\mathrm{G}=9.7 \%$ ), a common feature of insect mitochondrial DNA (Simon et al., 1994). Base frequencies were homogeneous among all sequences $\left(\chi^{2}=37.7, \mathrm{df}=288, P=\right.$ $1.0)$ and the overall transition/transversion bias was $R=$ 1.02. According to the guidelines given in Kumar et al. (1994) this value allowed us to use the uncorrected $p$-distance in further analyses, such as, the estimates of intraspecific and interspecific divergence and phylogenetic tree construction. 
TABLE 1. List of the species of Adelgidae sampled (in alphabetical order) and the Phylloxeridae outgroup species.

\begin{tabular}{|c|c|c|c|c|c|c|}
\hline Species & Sample No. & $\begin{array}{c}\text { GenBank } \\
\text { accession no. }\end{array}$ & Collection host plant & Locality & Date & Conservation \\
\hline Adelges laricis (Vallot, 1836) & 10555 & GU570998 & Picea abies (L.) H.Karst. & Buchlovice & 5.6 .2008 & kept at $-70^{\circ} \mathrm{C}$ \\
\hline Adelges laricis (Vallot, 1836) & 11500 & GU570999 & Picea abies (L.) H.Karst. & $\begin{array}{l}\text { České Budě- } \\
\text { jovice, Borek }\end{array}$ & 13.8.2008 & ditto \\
\hline Adelges laricis (Vallot, 1836) & 10960 & GU571000 & Picea abies (L.) H.Karst. & Nový Dvưr & 4.6.2008 & ditto \\
\hline Adelges laricis (Vallot, 1836) & 11031 & GU571001 & Picea abies (L.) H.Karst. & Nový Dvůr & 4.6.2008 & ditto \\
\hline Adelges tardus (Dreyfus, 1888) & P2554 & GU571002 & Picea abies (L.) H.Karst. & Nový Dvůr & 7.6.2007 & pure ethanol \\
\hline Adelges tardus (Dreyfus, 1888) & P1283-P1287 & $\begin{array}{l}\text { GU571003- } \\
\text { GU571007 }\end{array}$ & Picea abies (L.) H.Karst. & $\begin{array}{c}\text { České } \\
\text { Budějovice }\end{array}$ & 8.6 .2007 & ditto \\
\hline Adelges tardus (Dreyfus, 1888) & 10688 & GU571008 & Picea abies (L.) H.Karst. & Nový Dvůr & 4.6.2008 & kept at $-70^{\circ} \mathrm{C}$ \\
\hline Adelges tardus (Dreyfus, 1888) & 11458 & GU571009 & Picea abies (L.) H.Karst. & $\begin{array}{l}\text { České Budějo- } \\
\text { vice, Stromovka }\end{array}$ & 3.8 .2008 & ditto \\
\hline Adelges tardus (Dreyfus, 1888) & 11196 & GU571010 & Picea abies (L.) H.Karst. & $\begin{array}{l}\text { České Budějo- } \\
\text { vice, Stromovka }\end{array}$ & 3.8 .2008 & ditto \\
\hline Adelges tardus (Dreyfus, 1888) & 11340 & GU571011 & Picea abies (L.) H.Karst. & Praha, Břevnov & 10.8 .2008 & ditto \\
\hline $\begin{array}{l}\text { Aphrastasia pectinatae } \\
\text { (Cholodkovsky, 1888) }\end{array}$ & 3345 & GU571012 & $\begin{array}{l}\text { Abies concolor } \\
\text { Lindl. \& Gord. }\end{array}$ & $\begin{array}{l}\text { Vilnius } \\
\text { (Lithuania) }\end{array}$ & 31.7.2008 & pure ethanol \\
\hline $\begin{array}{l}\text { Cholodkovskya viridana } \\
\text { (Cholodkovsky, 1896) }\end{array}$ & $3326-3328$ & $\begin{array}{l}\text { GU571013- } \\
\text { GU571015 }\end{array}$ & Larix kaempferi Fortune & Nový Dvůr & 16.7.2008 & ditto \\
\hline $\begin{array}{l}\text { Dreyfusia nordmannianae } \\
\text { (Eckstein, 1890) }\end{array}$ & P1405 & GU571016 & Abies alba Mill. & Chvalčov & 13.4.2007 & ditto \\
\hline $\begin{array}{l}\text { Dreyfusia nordmannianae } \\
\text { (Eckstein, 1890) }\end{array}$ & $10540-10541$ & $\begin{array}{l}\text { GU571017- } \\
\text { GU571018 }\end{array}$ & Abies alba Mill. & Libín & 11.3.2008 & kept at $-70^{\circ} \mathrm{C}$ \\
\hline $\begin{array}{l}\text { Dreyfusia nordmannianae } \\
\text { (Eckstein, 1890) }\end{array}$ & $12009-12011$ & $\begin{array}{l}\text { GU571019- } \\
\text { GU571021 }\end{array}$ & $\begin{array}{l}\text { Picea orientalis (L.) } \\
\text { Peterm. }\end{array}$ & Buchlovice & 15.8 .2008 & ditto \\
\hline Dreyfusia piceae (Ratzeburg, 1844) & $3498-3499$ & $\begin{array}{l}\text { GU571022- } \\
\text { GU571023 }\end{array}$ & Abies alba Mill. & $\begin{array}{l}\text { Bystřice pod } \\
\text { Hostýnem }\end{array}$ & 17.9.2008 & pure ethanol \\
\hline Dreyfusia piceae (Ratzeburg, 1844) & 3622 & GU571024 & Abies alba Mill. & $\begin{array}{l}\text { České Budějo- } \\
\text { vice, Branišov }\end{array}$ & 9.11 .2008 & ditto \\
\hline Dreyfusia prelli (Grossmann, 1935) & P812, P814 & $\begin{array}{l}\text { GU571025, } \\
\text { GU571026 }\end{array}$ & $\begin{array}{l}\text { Picea orientalis (L.) } \\
\text { Peterm. }\end{array}$ & Buchlovice & 28.6.2005 & ditto \\
\hline Dreyfusia prelli (Grossmann, 1935) & P707 & GU571027 & $\begin{array}{l}\text { Picea orientalis (L.) } \\
\text { Peterm. }\end{array}$ & Nový Dvůr & 29.6.2005 & ditto \\
\hline Dreyfusia prelli (Grossmann, 1935) & P1963, P2693 & $\begin{array}{l}\text { GU571028, } \\
\text { GU571029 }\end{array}$ & $\begin{array}{l}\text { Picea orientalis (L.) } \\
\text { Peterm. }\end{array}$ & Buchlovice & 17.7.2007 & ditto \\
\hline Dreyfusia prelli (Grossmann, 1935) & P2633 & GU571030 & $\begin{array}{l}\text { Picea orientalis (L.) } \\
\text { Peterm. }\end{array}$ & Lednice & 28.6.2007 & ditto \\
\hline Dreyfusia prelli (Grossmann, 1935) & $10841-10843$ & $\begin{array}{l}\text { GU571031- } \\
\text { GU571033 }\end{array}$ & $\begin{array}{l}\text { Picea orientalis (L.) } \\
\text { Peterm. }\end{array}$ & Buchlovice & 20.6.2008 & kept at $-70^{\circ} \mathrm{C}$ \\
\hline Eopineus strobi (Hartig, 1837) & P2462-P2463 & $\begin{array}{l}\text { GU571034- } \\
\text { GU571035 }\end{array}$ & Pinus strobus $\mathrm{L}$. & Nový Dvůr & 24.5.2007 & pure ethanol \\
\hline Eopineus strobi (Hartig, 1837) & P2217 & GU571036 & Pinus strobus $\mathrm{L}$. & $\begin{array}{c}\text { České } \\
\text { Budějovice }\end{array}$ & 22.2.2007 & ditto \\
\hline Eopineus strobi (Hartig, 1837) & P2457-P2458 & $\begin{array}{l}\text { GU571037- } \\
\text { GU571038 }\end{array}$ & Pinus strobus $\mathrm{L}$. & $\begin{array}{c}\text { České } \\
\text { Budějovice }\end{array}$ & 22.5.2007 & ditto \\
\hline Eopineus strobi (Hartig, 1837) & 3098 & GU571039 & Pinus strobus L. & Nový Dvůr & 21.5.2008 & ditto \\
\hline Gilletteella cooleyi (Gillette, 1907) & P1740-P1743 & $\begin{array}{l}\text { GU571040- } \\
\text { GU571043 }\end{array}$ & $\begin{array}{l}\text { Pseudotsuga menziesii } \\
\text { (Mirb.) Franco }\end{array}$ & $\begin{array}{c}\text { České } \\
\text { Budějovice }\end{array}$ & 26.3.2007 & ditto \\
\hline Gilletteella cooleyi (Gillette, 1907) & $11009-11011$ & $\begin{array}{c}\text { GU571044- } \\
\text { GU571046 }\end{array}$ & Picea pungens Engelm. & $\begin{array}{l}\text { České } \\
\text { Budějovice }\end{array}$ & 30.6 .2008 & kept at $-70^{\circ} \mathrm{C}$ \\
\hline Gilletteella cooleyi (Gillette, 1907) & $11026-11028$ & $\begin{array}{l}\text { GU571047- } \\
\text { GU571049 }\end{array}$ & Picea pungens Engelm. & Praha & 21.6.2008 & ditto \\
\hline Gilletteella coweni (Gillette, 1907) & 3229 & GU571050 & $\begin{array}{l}\text { Pseudotsuga menziesii } \\
\text { (Mirb.) Franco }\end{array}$ & Praha, Břevnov & 22.6.2008 & pure ethanol \\
\hline Gilletteella coweni (Gillette, 1907) & $3579 \mathrm{a}$ & GU571051 & $\begin{array}{l}\text { Pseudotsuga menziesii } \\
\text { (Mirb.) Franco }\end{array}$ & $\begin{array}{l}\text { Stráž nad } \\
\text { Nežárkou }\end{array}$ & 12.2.2008 & ditto \\
\hline
\end{tabular}


TABLE 1 (continued).

\begin{tabular}{|c|c|c|c|c|c|c|}
\hline Species & Sample No. & $\begin{array}{c}\text { GenBank } \\
\text { accession no. }\end{array}$ & Collection host plant & Locality & Date & Conservation \\
\hline $\begin{array}{l}\text { Gilletteella coweni } \\
\text { (Gillette, 1907) }\end{array}$ & 3582 & GU571052 & $\begin{array}{l}\text { Pseudotsuga menziesii } \\
\text { (Mirb.) Franco }\end{array}$ & $\begin{array}{l}\text { Stráž nad } \\
\text { Nežárkou }\end{array}$ & 28.10 .2008 & ditto \\
\hline $\begin{array}{l}\text { Sacchiphantes abietis } \\
\text { (Ratzeburg, } 1843\end{array}$ & P1044 & GU571053 & Picea abies (L.) H.Karst. & Zdiměřice & 24.8 .2005 & ditto \\
\hline $\begin{array}{l}\text { Sacchiphantes abietis } \\
\text { (Linnaeus, 1758) }\end{array}$ & P2416-P2420 & $\begin{array}{l}\text { GU571054- } \\
\text { GU571058 }\end{array}$ & Picea abies (L.) H.Karst. & Troják & 27.4.2007 & ditto \\
\hline $\begin{array}{l}\text { Sacchiphantes abietis } \\
\text { (Linnaeus, 1758) }\end{array}$ & P2887 & GU571059 & Picea abies (L.) H.Karst. & Čertovo jezero & 20.8 .2007 & ditto \\
\hline $\begin{array}{l}\text { Sacchiphantes abietis } \\
\text { (Linnaeus, 1758) }\end{array}$ & 11624 & GU571060 & Picea abies (L.) H.Karst. & $\begin{array}{l}\text { Šumava Mts, } \\
\text { Jenišov }\end{array}$ & 24.8 .2008 & kept at $-70^{\circ} \mathrm{C}$ \\
\hline $\begin{array}{l}\text { Sacchiphantes abietis } \\
\text { (Linnaeus, 1758) }\end{array}$ & 11697 & GU571061 & Picea abies (L.) H.Karst. & $\begin{array}{l}\text { Šumava Mts, } \\
\text { Vítkův Kámen }\end{array}$ & 27.8.2008 & ditto \\
\hline $\begin{array}{l}\text { Sacchiphantes viridis } \\
\text { (Ratzeburg, 1843) }\end{array}$ & P846 & GU571062 & Picea abies (L.) H.Karst. & Český Krumlov & 25.7.2005 & pure ethanol \\
\hline $\begin{array}{l}\text { Sacchiphantes viridis } \\
\text { (Ratzeburg, 1843) }\end{array}$ & 11380 & GU571063 & Picea abies (L.) H.Karst. & $\begin{array}{l}\text { České Budějovice, } \\
\text { Haklovy Dvory }\end{array}$ & 8.8.2008 & kept at $-70^{\circ} \mathrm{C}$ \\
\hline $\begin{array}{l}\text { Sacchiphantes viridis } \\
\text { (Linnaeus, 1758) }\end{array}$ & 11350 & GU571064 & Picea abies (L.) H.Karst. & $\begin{array}{l}\text { Kapaonik Natl. Park } \\
\text { (Serbia) }\end{array}$ & 8.8 .2008 & ditto \\
\hline $\begin{array}{l}\text { Sacchiphantes viridis } \\
\text { (Ratzeburg, 1843) }\end{array}$ & 11362 & GU571065 & Picea abies (L.) H.Karst. & Lednice & 13.8 .2008 & ditto \\
\hline $\begin{array}{l}\text { Sacchiphantes viridis } \\
\text { (Ratzeburg, 1843) }\end{array}$ & 11550 & GU571066 & Picea abies (L.) H.Karst. & Strmilov & 13.8 .2008 & ditto \\
\hline $\begin{array}{l}\text { Sacchiphantes viridis } \\
\text { (Ratzeburg, 1843) }\end{array}$ & 11573 & GU571067 & Picea abies (L.) H.Karst. & Želetava & 13.8 .2008 & ditto \\
\hline $\begin{array}{l}\text { Sacchiphantes viridis } \\
\text { (Ratzeburg, 1843) }\end{array}$ & 11619 & GU571068 & Picea abies (L.) H.Karst. & Fulnek & 14.8.2008 & ditto \\
\hline $\begin{array}{l}\text { Pineus cembrae } \\
\text { (Cholodkovsky, 1888) }\end{array}$ & 10536 & GU571069 & Pinus cembra $\mathrm{L}$. & Těchobuz u Pacova & 22.4 .2008 & pure ethanol \\
\hline $\begin{array}{l}\text { Pineus cembrae } \\
\text { (Cholodkovsky, 1888) }\end{array}$ & $3071-3072$ & $\begin{array}{l}\text { GU571070- } \\
\text { GU571071 }\end{array}$ & Pinus cembra $\mathrm{L}$. & Těchobuz u Pacova & 22.4 .2008 & ditto \\
\hline $\begin{array}{l}\text { Pineus cembrae } \\
\text { (Cholodkovsky, 1888) }\end{array}$ & 3497 & GU571072 & Pinus cembra $\mathrm{L}$. & Těchobuz u Pacova & 7.9.2008 & ditto \\
\hline $\begin{array}{l}\text { Pineus cembrae } \\
\text { (Cholodkovsky, 1888) }\end{array}$ & 3583 & GU571073 & Pinus cembra $\mathrm{L}$. & Buchlovice & 30.10 .2008 & ditto \\
\hline $\begin{array}{l}\text { Pineus orientalis } \\
\text { (Dreyfus, 1889) }\end{array}$ & P2486-2488 & $\begin{array}{l}\text { GU571074- } \\
\text { GU571076 }\end{array}$ & $\begin{array}{l}\text { Picea orientalis (L.) } \\
\text { Peterm. }\end{array}$ & Buchlovice & 23.5.2007 & ditto \\
\hline $\begin{array}{l}\text { Pineus orientalis } \\
\text { (Dreyfus, 1889) }\end{array}$ & $10580-10582$ & $\begin{array}{l}\text { GU571077- } \\
\text { GU571079 }\end{array}$ & $\begin{array}{l}\text { Picea orientalis (L.) } \\
\text { Peterm. }\end{array}$ & Lednice & 3.6 .2008 & kept at $-70^{\circ} \mathrm{C}$ \\
\hline $\begin{array}{l}\text { Pineus orientalis } \\
\text { (Dreyfus, 1889) }\end{array}$ & $10655-10657$ & $\begin{array}{l}\text { GU571080- } \\
\text { GU571082 }\end{array}$ & $\begin{array}{l}\text { Picea orientalis (L.) } \\
\text { Peterm. }\end{array}$ & Buchlovice & 5.6 .2008 & ditto \\
\hline $\begin{array}{l}\text { Pineus pineoides } \\
\text { (Cholodkovsky, 1903) }\end{array}$ & 3487 & GU571083 & Picea abies (L.) H.Karst. & $\begin{array}{l}\text { Šumava Mts, } \\
\text { Jenišov }\end{array}$ & 24.8.2008 & pure ethanol \\
\hline Pineus pini (Macquart, 1819) & P2269 & GU571084 & Pinus sylvestris $\mathrm{L}$. & Praha, Břevnov & 4.4.2007 & ditto \\
\hline Pineus pini (Macquart, 1819) & P2265 & GU571085 & Pinus sylvestris L. & Praha, Břevnov & 4.4.2007 & ditto \\
\hline Pineus pini (Macquart, 1819) & P2353 & GU571086 & Pinus sylvestris L. & $\begin{array}{l}\text { Bystřice pod } \\
\text { Hostýnem }\end{array}$ & 25.4 .2007 & ditto \\
\hline Pineus pini (Macquart, 1819) & $3230-3231$ & $\begin{array}{l}\text { GU571087- } \\
\text { GU571088 }\end{array}$ & Pinus sylvestris L. & Praha, Břevnov & 22.6 .2008 & ditto \\
\hline Pineus pini (Macquart, 1819) & 3576 & GU571089 & Pinus sylvestris L. & České Budějovice & 10.11 .2008 & ditto \\
\hline Viteus vitifoliae (Fitch, 1855) & $12215-12217$ & $\begin{array}{l}\text { GU571090- } \\
\text { GU571091 }\end{array}$ & Vitis vinifera $\mathrm{L}$. & Polešovice & 17.9.2008 & kept at $-70^{\circ} \mathrm{C}$ \\
\hline $\begin{array}{l}\text { Phylloxera coccinea (von } \\
\text { Heyden, 1837) }\end{array}$ & $\begin{array}{c}3205 \\
3224-3225\end{array}$ & $\begin{array}{l}\text { GU571092 } \\
\text { GU571093 } \\
\text { GU571094 }\end{array}$ & Quercus robur L. & Lednice & 17.6.2008 & pure ethanol \\
\hline
\end{tabular}

The phylogenetic analysis showed that all genera formed monophyletic groups. Since the topology of the trees generated by different methods was similar, only the
NJ and Bayesian trees are presented (Fig. 1). The only difference between the NJ and the Bayesian trees is the position of the Pineus/Eopineus cluster. While in the NJ 
$\square$ Dreyfusia prelli (P812) ] prelli

Dreyfusia prelli (P707)

Dreyfusia prelli (814) prelli Dreyfusia prelli (814) prelli

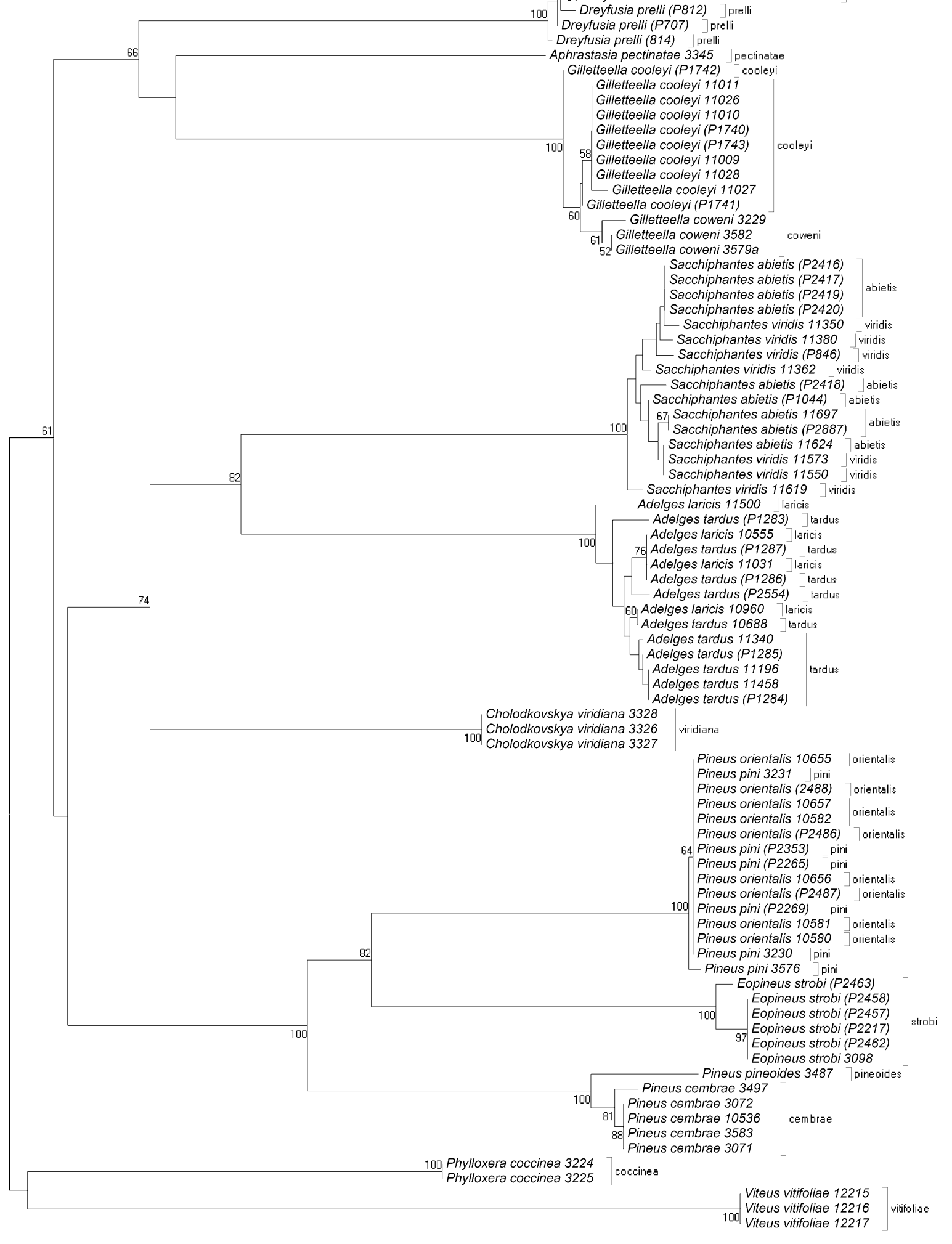




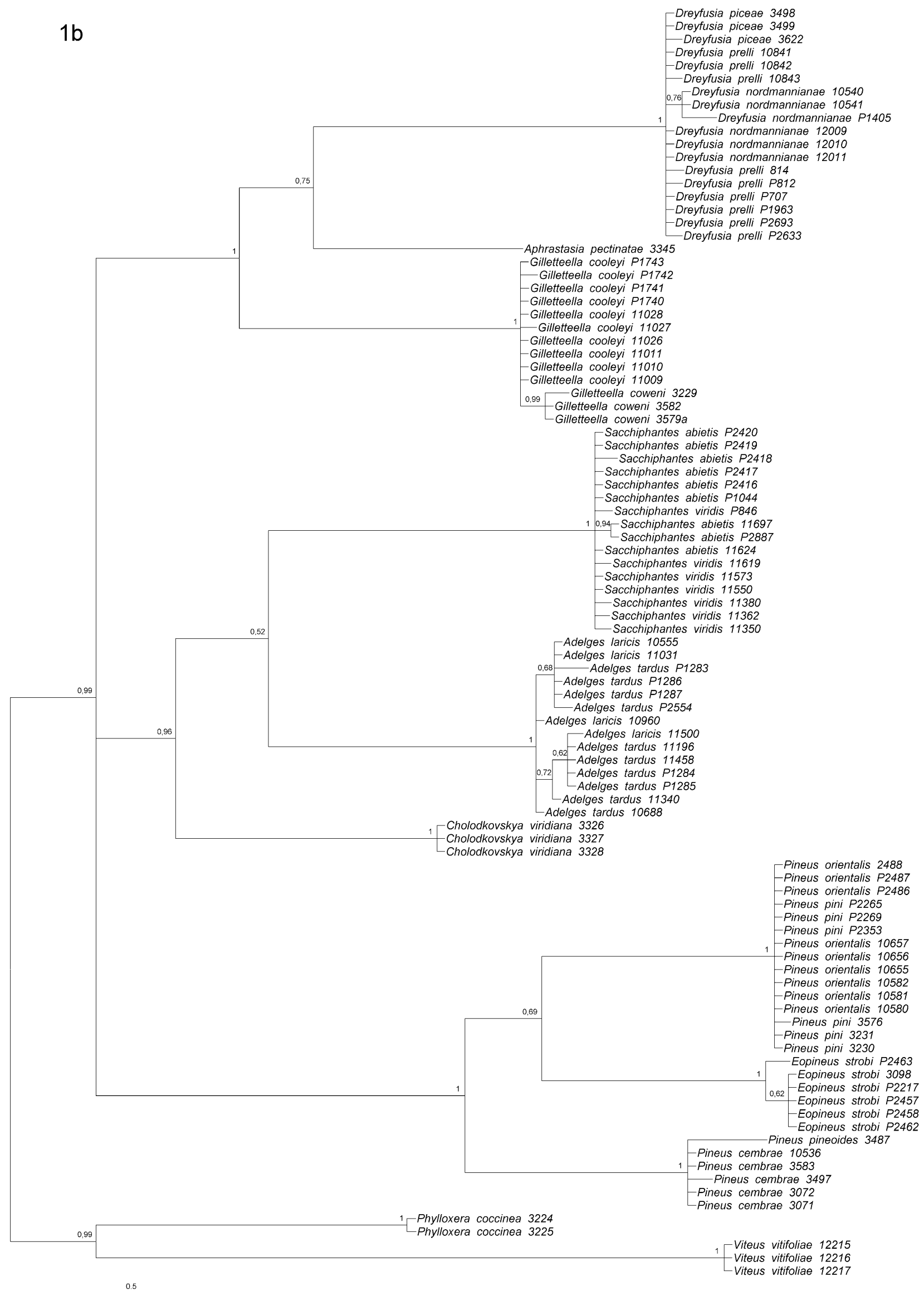

Fig. 1. Evolutionary relationships of 97 specimens representing 16 species of adelgids and 2 phylloxerids. 1a - Neighbour-Joining tree, the bootstrap values (10000 replicates) are shown next to the branches; $1 \mathrm{~b}$ - Phylogram from the MrBayes analysis. Numbers above each node represent the posterior probability support. 
TABLE 2. Intraspecific and interspecific congeneric distances (uncorrected $p$-distance) for 16 species of adelgids.

\begin{tabular}{|c|c|c|c|c|c|}
\hline \multirow{2}{*}{ Species } & \multirow{2}{*}{$\begin{array}{c}\text { No. of } \\
\text { specimens }\end{array}$} & \multicolumn{2}{|c|}{ Intraspecific divergence $(\%)$} & \multicolumn{2}{|c|}{ Interspecific congeneric divergence $(\%)$} \\
\hline & & Mean & Range & Mean & Range \\
\hline Dreyfusia prelli & 9 & 0.14 & $0.0-0.32$ & & \\
\hline Dreyfusia nordmannianae & 6 & 0.24 & $0.0-0.61$ & Dreyfusia & \\
\hline Dreyfusia piceae & 3 & 0.10 & $0.0-0.15$ & 0.18 & $0.0-0.76$ \\
\hline Aphrastasia pectinatae & 1 & $\mathrm{n} / \mathrm{c}$ & & & \\
\hline Gilletteella coweni & 3 & 0.21 & $0.0-0.31$ & Gilletteela & \\
\hline Gilletteella cooleyi & 10 & 0.06 & $0.0-0.32$ & 0.20 & $0.0-0.76$ \\
\hline Sacchiphantes abietis & 9 & 0.21 & $0.0-0.46$ & Sacchiphantes & \\
\hline Sacchiphantes viridis & 7 & 0.35 & $0.0-0.48$ & 0.28 & $0.0-0.62$ \\
\hline Adelges laricis & 4 & 0.43 & $0.0-0.76$ & Adelges & \\
\hline Adelges tardus & 10 & 0.38 & $0.0-0.94$ & 0.38 & $0.0-1.10$ \\
\hline Cholodkovskya viridiana & 3 & 0.00 & 0.00 & & \\
\hline Pineus orientalis & 9 & 0.00 & 0.00 & & \\
\hline Pineus pini & 6 & 0.05 & $0.0-0.15$ & & \\
\hline Eopineus strobi & 6 & 0.15 & $0.0-0.46$ & & \\
\hline Pineus pineoides & 1 & $\mathrm{n} / \mathrm{c}$ & & Pineus & \\
\hline Pineus cembrae & 5 & 0.12 & $0.0-0.30$ & 4.12 & $0.0-7.61$ \\
\hline Phylloxera coccinea & 2 & 0.00 & 0.00 & & \\
\hline Viteus vitifoliae & 3 & 0.00 & 0.00 & & \\
\hline
\end{tabular}

$\mathrm{n} / \mathrm{c}$ - evolutionary distances not computed

tree Pineus/Eopineus stands as a sister group to the Sacchiphantes/Adelges/Cholodkovskya cluster (Fig. 1a), in the Bayesian tree it forms a polytomy with the branches leading to the Giletteella/Dreyfusia and Sacchiphantes/Adelges/Cholodkovskya clusters (Fig. 1b). Note that the bootstrap support for such clustering on the NJ tree is very low (21\%).

Most clusters were strongly supported and formed either by single species (e.g. Cholodkovskya) or by species complexes (Adelges, Sacchiphantes, Dreyfusia, Gilletteella, and Pineus pini/orientalis). Eopineus is nested within Pineus and should thus either be synonymized or three genera have to be recognized in this cluster. On the other hand, nodes representing the subfamilies sensu Annand (1928), Börner \& Heinze (1957) or Steffan (1968) were not monophyletic.

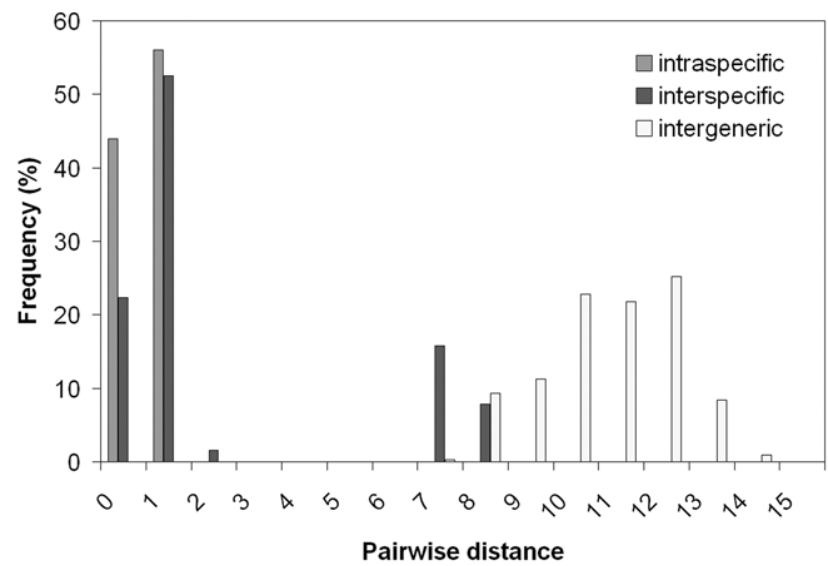

Fig. 2. Overlap of the intraspecific, interspecific congeneric, and intergeneric genetic variability ( $p$-distance uncorrected).
The mean intraspecific divergence for all adelgid species was $0.15 \%$ (range $0.00-0.76 \%$ ). Mean intraspecific divergence was 0.38 (range 0.00-1.10\%) for the Adelges complex (laricis/tardus), 0.3 (range $0.00-0.62 \%$ ) for the Sacchiphantes complex (abietis/viridis), and 0.02 (range 0.00-0.15) for the Pineus complex (orientalis/pini). The mean interspecific divergence was $8.23 \%$ (range $0.00-13.24 \%$ ) for all adelgid species. However, the interspecific congeneric divergence was much lower, ranging from 0.0 to $4.12 \%$ (Table 2). There was a considerable overlap of the intraspecific and interspecific congeneric divergencies (Table 2), as can be seen also on the histogram in Fig. 2. On the other hand, there is no overlap with the divergencies at the generic level (Table 3 and Fig. 2), with the mean value being 10.26\% (range 7.00-13.24\%).

The threshold for species identification evaluated by TaxonDNA was $0.6 \%$, nevertheless, only $41(44.56 \%)$ of the sequences were correctly identified according to the "Best Match" criteria; 42 sequences were ambiguous $(45.65 \%)$ and $9(9.78 \%)$ were incorrectly identified (including 2 species represented by only a single specimen). The higher threshold values (at 1\%,2\% and $3 \%$ levels) were also tested as recommended by Meier et al. (2008) and Ratnasingham \& Hebert (2007). As expected, these values did not increase the identification rate, which remained the same as at the $0.6 \%$ level in all these tests.

\section{DISCUSSION AND CONCLUSIONS}

Our results indicate that "DNA barcoding" using the COI gene can successfully identify adelgid species that are clearly delineated by classical taxonomy. Molecular data produced monophyletic groups representing single 
TABLE 3. Estimates of evolutionary divergence (uncorrected $p$-distance) among genera.

\begin{tabular}{lccccccc}
\hline & Dreyfusia & Aphrastasia & Gilletteella & Sacchiphantes & Cholodkovskya & Adelges & Pineus/Eopineus \\
\hline Dreyfusia & & 0.078 & 0.083 & 0.107 & 0.088 & 0.104 & 0.109 \\
Aphrastasia & 0.010 & & 0.071 & 0.092 & 0.091 & 0.083 & 0.104 \\
Gilletteella & 0.010 & 0.010 & & 0.095 & 0.081 & 0.097 & 0.119 \\
Sacchiphantes & 0.012 & 0.011 & 0.011 & & 0.077 & 0.079 & 0.120 \\
Cholodkovskya & 0.011 & 0.011 & 0.010 & 0.010 & & 0.078 & 0.097 \\
Adelges & 0.011 & 0.011 & 0.011 & 0.010 & 0.010 & & 0.103 \\
Pineus/Eopineus & 0.011 & 0.011 & 0.012 & 0.011 & 0.010 & 0.010 & \\
\hline
\end{tabular}

The number of base differences per site obtained by averaging over all sequence pairs between genera is shown above diagonal. All results are based on the pairwise analysis of 97 sequences. Standard error estimates are shown in the lower-left part of the matrix and were obtained by a bootstrap procedure (1000 replicates).

genera. However, this marker was not sufficient to distinguish morphologically identical species in species complexes, whose description is based on ecology.

Mean values of overall intraspecific $(0.15 \%)$ and interspecific $(8.23 \%)$ divergence are comparable to those of other insects, such as $0.46 \%$ and $4.41-6.02 \%$ reported for tropical Lepidoptera (Hajibabaei et al., 2006) or $0.17 \%$ and $5.78 \%$ for parasitoid flies (Smith et al., 2006). They are also comparable to the results obtained by Havill et al. (2007) and Foottit et al. (2009), although direct comparison is difficult since these authors use a different generic taxonomy and the species sampled only partially overlapped. In addition, figures in the Foottit et al. (2009) study are also higher because the extensive sampling of two pest species suggested the existence of two or three cryptic species.

According to the guidelines proposed by Hebert et al. (2004), successful species identification is possible if mean interspecific divergence equals $10 \times$ the mean intraspecific divergence, and in an ideal case, there should be an observable "barcoding gap", that is, a separation between mean intra- and interspecific congeneric $\mathrm{COI}$ sequences (Meyer \& Paulay, 2005). However, it is argued by Meier et al. (2006) that instead of the mean value, only the smallest interspecific distance should be used. Although the observed difference in the mean values for our data set fulfills the first criterion, it is not so for the other two. The biggest issue is the large overlap of intraand interspecific divergence, which is most likely caused by the inclusion of species complexes. But there are examples of interspecific congeneric distances as low as 0.001 for the well defined species $D$. prelli and D. piceae, which makes the use of "DNA barcoding" doubtful. Since the evolution of the species complexes is recent, it is likely that the $C O I$ locus is not the best marker for individual species identification.

Our analysis supports the recognition of eight genera as proposed by Börner \& Heinze (1957) or Steffan (1968) rather then the two genera system of Annand (1928). Phylogenetic trees distinguished most genera very clearly. However, our results differ from Havill et al. (2007). The first difference is the placement of the Pineus cluster, which is not distinctly separated from all the other adelgids. In our NJ tree and Bayesian analysis, it is positioned closer to the Adelges and Sacchiphantes groups. Second, both trees support sister group relationships between Gil- letteella and Dreyfusia, while Havill et al. (2007) placed Gilletteella closer to the Sacchiphantes/Adelges group. Taken together, our data best fit the system presented by Börner \& Heinze (1957).

The most problematic group appears to be Dreyfusia. The status of its species has been disputed. Mantovani et al. (2001) conclude, based on mitochondrial DNA sequences, that at least three of the five Dreyfusia species known from conifers in Italy are doubtful. Our results are similar. Although some species form a species complex (nordmannianae/piceae), the observed pattern is striking. D. prelli is very distinct, based on morphology and life cycle characteristics. It is therefore quite surprising that the DNA marker did not reveal any differences with respect to the other species.

On the other hand, two sister species, G. cooleyi and G. coweni, can be identified based on the 3rd codon position difference (one silent substitution). Adding more sympatric samples from different localities is in this case highly desirable, as well as using other, more quickly evolving markers.

In conclusion, Adelgidae are another group for which the "DNA barcoding"is not the tool of first choice for species identification, although it can provide helpful suggestions for the identification of species at the genus level. The main problem is that the intraspecific and interspecific congeneric variability do not form two separate intervals with a distinct "barcoding gap". In addition, several species share the same haplotypes, thus the identification of these species is impossible. Furthermore, it is suggested that the eight genus system (with revision of the Pineus genus), previously proposed based on morphological studies, should continue to be used. Species complexes still remain an interesting puzzle both at the ecological and genetic level, and as suggested by Foottit et al. (2009) further studies are needed to resolve their species status.

ACKNOWLEDGEMENTS. We thank A. Tatarenkov for comments on an early version of this manuscript. We also thank two anonymus reviewers and R. Meier for valuable advice, which greatly improved the manuscript. The technical assistance of $\mathrm{V}$. Chalušová (Institute of Entomology, BC ASCR České Budějovice, Czech Republic) is greatly appreciated. This work was funded by Grant No. A600960705 of the Grant Agency of the Academy of Sciences of the Czech Republic (Prague) and from the Institute of Entomology Z50070508. L.K. acknowledges that 
her Ph.D. study was funded by grant 521/08/H042 from the Grant Agency of the Czech Republic, Prague.

\section{REFERENCES}

Annand P.N. 1928: A Contribution Toward a Monograph of the Adelginae (Phylloxeridae) of North America. Stanford Univ. Press, Palo Alto, CA, 146 pp.

BinAzZI A. 2000: Notes on and key to winged forms of adelgids recorded from Italy (Homoptera, Aphidoidea, Adelgidae). Redia 83: 187-215.

BinazZI A. \& Covassi M. 1991: Contributions to the knowledge of the conifer aphid fauna XII, a review of the species of Dreyfusia Boerner occurring in Italy with description of Dreyfusia nebrodensis n. sp. (Homoptera: Adelgidae). Redia 74: $233-288$.

Blackman R.L. \& Eastop V.F. 1994: Aphids on the World's Trees: An Identification and Information Guide. CABI, Wallingford, $987 \mathrm{pp}$.

Bodenheimer F.S. \& Swirski E. 1957: Aphidoidea of the Middle East. The Weizmann Science Press of Israel, Jerusalem, 378 pp.

BöRNER C.V. 1908: Eine monographische Studie über die Chermiden. Arb. Kais. Biol. Anst. Land. Forstw. 6: 81-320.

BöRNER C.V. 1930: Beiträge zur einem neuen System der Blattläuse. Arch. Klassif. Phylogen. Entomol. 50: 115-194.

BÖRNER C. 1952: Europae centralis Aphides, die Blattläuse Mitteleuropas. Mitt. Thurin. Bot. Ges. 3: 1-484.

Börner C.V. \& Heinze K. 1957: Aphidina - Aphidoidea. In Blunck H. (ed.): Tierische Schädlinge an Nutzpflanzen. Homoptera. 2. Teil. Paul Parey, Berlin, pp. 323-355.

CARTER C.I. 1971: Conifer Woolly Aphids (Adelgidae) in Britain. Forestry Commission Bulletin No. 42, London, 51 pp.

Cholodkovsky N. 1896: Zur Biologie der Lärchen-ChermesArten. Zool. Anz. 19: 37-40.

Dmitriev G.V. 1965: Chermesinae - pests of coniferous trees of Ukraine. In: Nature Conservation. Urozhai, Kiev, pp. 157-203.

Folmer O., Black M., Hoeh W., Lutz R. \& Vrijenhoek R. 1994: DNA primers for amplification of mitochondrial cytochrome $c$ oxidase subunit I from diverse metazoan invertebrates. Mol. Marine Biol. Biotechnol. 3: 294-299.

Foottit R.G., Maw E.L., Havill N.P., Ahern R.G. \& MontGOMERY M.E. 2009: DNA barcodes to identify species and explore diversity in the Adelgidae (Insecta: Hemiptera: Aphidoidea). Mol. Ecol. Res. 9: 188-195.

Frati F., Spinsanti G. \& Dallai R. 2001: Genetic variation of $\mathrm{mt}$ COII gene in the collembolan Isotoma klovstadi from Victoria land, Antarctica: evidence for population differentiation. Polar Biol. 24: 934-940.

Guindon S. \& Gascuel O. 2003: A simple, fast, and accurate algorithm to estimate large phylogenies by maximum likelihood. Syst. Biol. 52: 696-704.

Hajibabaei M., Janzen D.H., Burns J.M., Hallwachs W. \& HeBert P.D.N. 2006: DNA barcodes distinguish species of tropical Lepidoptera. PNAS 103: 968-977.

Havill N.P. \& Foottit R.G. 2007: Biology and evolution of Adelgidae. Annu. Rev. Entomol. 52: 325-349.

Havill N.P., Foottit R.G. \& von Dohlen C.D. 2007: Evolution of host specialization in the Adelgidae (Insecta: Hemiptera) inferred from molecular phylogenetics. Mol. Phylog. Evol. 44: 357-370.

Hebert P.D.N., Penton E.H., Burns J.M., Janzen D.H. \& HallWACHS W. 2004: Ten species in one: DNA barcoding reveals cryptic species in the neotropical skipper butterfly Astraptes fulgerator. PNAS 101: 14812-14817.

HeInZE K. 1962: Pflanzenschädliche Blattläusarten der Familien Lachnidae, Adelgidae und Phylloxeridae, eine systematischfaunistische Studie. Dt. Entomol. Z. 9: 143-227.

INOUYE M. 1945: Monographische Studie über die japanischen Koniferen-Gallenläuse (Adelgidae). Bull. Sapporo Bran. Gov. For. Exp. Stn. 15: 1-91.

Kumar S., Tamura K. \& Nei M. 1994: MEGA: Molecular Evolutionary Genetics Analysis software for microcomputers. Comput. Appl. Biosci. 10: 189-191.

Lampel G. 1968: Die Biologie des Blattlaus-Generationswechsels. Gustav Fischer, Jena, 264 pp.

Mantovani B., Francardi V., Binazzi A. \& Leccese A. 2001: A molecular approach to differentiate the species of Dreyfusia Börner occurring in Italy (Aphidoidea, Adelgidae). Redia 84: 151-159.

Meier R., Kwong S., Vaidya G. \& NG P.K.L. 2006: DNA Barcoding and taxonomy in Diptera: a tale of high intraspecific variability and low identification success. Syst. Biol. 55: 715-728.

Meier R., Zhang G. \& Farhan A. 2008: The use of mean instead of smallest interspecific distances exaggerates the size of the "barcoding gap" and leads to misidentification. Syst. Biol. 57: 809-813.

Meyer C.P. \& Paulay G. 2005: DNA barcoding: error rates based on comprehensive sampling. PLoS Biol. 3: 2229-2238.

Pashchenko N.F. 1988: Suborder Aphidinea, Aphids. In Leer P.A. (ed.): Keys to Insects of the Far East of USSR. Nauka, Leningrad, pp. 546-686 [in Russian].

PAŠEK V. 1954: Vošky našich lesných drevín. [Adelgids of our Coniferous Trees.] Veda, Bratislava, 319 pp.

Posada D. \& Crandall K.A. 1998: Modeltest: testing the model of DNA substitution. Bioinformatics 14: 817-818.

Ratnasingham S. \& Hebert P.D.N. 2007: BOLD: the Barcode of Life Data System. Mol. Ecol. Notes 7: 355-364.

Ronquist F. \& HuelsenBeCK J.P. 2003: MRBAYES 3: Bayesian phylogenetic inference under mixed models. Bioinformatics 19: $1572-1574$.

Shaposnikov G.CH. 1964: Aphidinea. In Bej-Bienko G.Ja. (ed.): Insects of the European Part of the USSR: Identification Key. Nauka, Moscow, pp. 489-616 [in Russian].

ShEARER T.L. \& COFFroth M.A. 2008: Barcoding corals: limited by interspecific divergence, not intraspecific variation. Mol. Ecol. Res. 8: 247-255.

Simon S., Frati F., Beckenbach A., Crespi B., Liu H. \& Flook P. 1994: Evolution, weighting, phylogenetic utility of mitochondrial gene sequences and compilation of conserved polymerase chain reaction primers. Ann. Entomol. Soc. Am. 87: 651-701.

Smith M.A., Woodley N.E., Janzen D.H., Hallwachs W. \& HEBERT P.D.N. 2006: DNA barcodes reveal cryptic hostspecificity within the presumed polyphagous members of a genus of parasitoid flies (Diptera: Tachinidae). PNAS 103: 3657-3662.

StefFan A.W. 1961: Die Stammes- und Siedlungsgeschichte des Artenkreises Sacchiphantes viridis (Ratzeburg, 1843) (Adelgidae, Aphidoidea). Zoologica 109: 1-113.

Steffan A.W. 1968: Evolution und Systematik der Adelgidae (Homoptera: Aphidina): Eine Verwandtschaftsanalyse auf vorwiegend ethologischer, zytologischer und karyologischer Grundlage. Zoologica 115: 1-139.

StefFAn A.W. 1972: Unterordnung Aphidina, Blattläuse. In Schwenke W. (ed.): Die Forstschädlinge Europas. Ein Handbuch in fünf Bänden. Parey, Hamburg, pp. 162-386. 
Swofrord D.L. 2003: PAUP*. Phylogenetic Analysis Using Parsimony (*and Other Methods). Version 4. Sinauer Associates, Sunderland, Massachusetts.

Szelegiewicz H. 1968: Mszyce, Aphidoidea. Katalog Fauny Polski 21: 1-316 [in Polish].

Tamura K., Dudley J., Nei M. \& Kumar S. 2007: MEGA 4: Molecular Evolutionary Genetics Analysis (MEGA) software version 4.0. Mol. Biol. Evol. 24: 1596-1599.

Tautz D., Arctander P., Minelli A., Thomas R.H. \& Vogler A.P. 2003: A plea for DNA taxonomy. Trends Ecol. Evol. 18: $70-74$.
Vences M., Thomas M., Bonett R.M. \& Vieites D.R. 2005: Deciphering amphibian diversity through DNA barcoding: chances and challenges. Phil. Trans. R. Soc. (B) 360: $1859-1868$

Wiemers M. \& Fiedler K. 2007: Does the DNA barcoding gap exist? - a case study in blue butterflies (Lepidoptera: Lycaenidae). Front. Zool. 4: 8.

Received October 1, 2009; revised and accepted January 4, 2010 